\title{
Electroacupuncture inhibits sodium nitroprusside-mediated chondrocyte apoptosis through the mitochondrial pathway
}

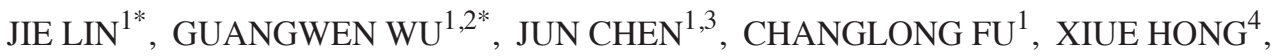 \\ $\mathrm{LI} \mathrm{LI}^{4}$, XIANXIANG LIU ${ }^{1}$ and MINGXIA WU ${ }^{4}$ \\ ${ }^{1}$ Academy of Integrative Medicine; ${ }^{2}$ Fujian Provincial Key Laboratory of Integrative Medicine on Geriatrics; \\ ${ }^{3}$ College of Integrative Medicine, Fujian University of Traditional Chinese Medicine, Fuzhou, Fujian 350122; \\ ${ }^{4}$ Department of Acupuncture and Moxibusion, The Second Affiliated People's Hospital of \\ Fujian University of Traditional Chinese Medicine, Fuzhou, Fujian 350003, P.R. China
}

Received February 15, 2018; Accepted August 22, 2018

DOI: $10.3892 / \mathrm{mmr} .2018 .9498$

\begin{abstract}
In China, electroacupuncture (EA) is a therapeutic method that is extensively applied in the clinical treatment of osteoarthritis (OA); however, the underlying molecular mechanism remains unclear. Chondrocyte apoptosis may be observed in cartilage tissue in $\mathrm{OA}$, and is often considered a key target for the treatment of this condition. Therefore, the present study aimed to determine the effects of EA on sodium nitroprusside (SNP)-induced chondrocyte apoptosis. Chondrocytes were obtained from the knee joints of Sprague Dawley rats by type II collagenase digestion. Following microscopic observation and authentication with type II collagen immunohistochemistry, articular cartilage cells were used in subsequent experiments. Using inverted phase contrast microscopy, DAPI staining and flow cytometry, it was revealed that chondrocytes treated with SNP became apoptotic, whereas EA inhibited SNP-induced chondrocyte apoptosis. Subsequently, JC-1 single staining, reverse transcription-quantitative polymerase chain reaction analysis, western blotting, colorimetric assays and immunofluorescence staining were performed for further investigation. The results demonstrated that, when compared with normal chondrocytes, the mitochondrial membrane potential of SNP-treated chondrocytes was markedly lowered, B-cell
\end{abstract}

Correspondence to: Professor Xianxiang Liu, Academy of Integrative Medicine, Fujian University of Traditional Chinese Medicine, 1 Qiuyang Road, Minhou, Fuzhou, Fujian 350122, P.R. China

E-mail: liuxianxiang@163.com

Professor Mingxia Wu, Department of Acupuncture and Moxibusion, The Second Affiliated People's Hospital of Fujian University of Traditional Chinese Medicine, 13 Hudong Road, Gulou, Fuzhou, Fujian 350003, P.R. China

E-mail:wumingxiafz@163.com

*Contributed equally

Keywords: electroacupuncture, osteoarthritis, sodium nitroprussideinduced chondrocyte apoptosis, molecular mechanism lymphoma 2 (Bcl-2) expression was reduced, and the expression levels of Bcl-2-associated $\mathrm{X}$ protein (Bax), cytochrome $c$, caspase- 9 and caspase- 3 were increased. Compared with in SNP-treated chondrocytes, the decrease in the mitochondrial membrane potential of chondrocytes treated with SNP and EA was smaller, Bcl-2 expression was increased, and the expression levels of Bax, cytochrome $c$, caspase- 9 and caspase-3 were decreased following EA intervention. In conclusion, the present study demonstrated that EA modulated the mitochondrial pathway to suppress SNP-mediated chondrocyte apoptosis. Therefore, EA may be of value in the treatment of OA.

\section{Introduction}

Osteoarthritis (OA) is a common chronic degenerative joint disease, clinically manifesting with pain, swelling and stiffness, and progressive OA may lead to permanent disability (1). The clinical manifestations of OA seriously affect the work and everyday life of patients, and may pose a major socioeconomic burden $(2,3)$. With the gradual increase in the size of the elderly population, the incidence of OA is also expected to increase (4). In order to alleviate the personal and social burden, it is particularly important to investigate safe and effective preventive and curative measures.

There is currently no particularly effective treatment for OA. Common treatment methods for the relief of symptoms, including non-steroidal anti-inflammatory drugs, hyaluronic acid injections and arthroplasty, among others, are associated with certain limitations (5-7). In China, in addition to the aforementioned methods, the treatment of OA also includes alternative methods, including Chinese herbal medicine, acupuncture and massage therapy, among which, electroacupuncture (EA) is often used in the management of OA. Certain clinical and evidence-based studies have suggested that EA can effectively relieve pain and improve joint function (8-10). It was previously demonstrated that EA promotes the differentiation of bone marrow-derived mesenchymal stem cells into chondrocytes (11), promotes the proliferation of chondrocytes (12), and reduces the inflammatory response of chondrocytes induced by tumor necrosis factor $\alpha$ (13). However, the precise mechanism of 
action of EA in the treatment of $\mathrm{OA}$ remains to be fully elucidated.

The principal pathological manifestations of OA are cartilage degeneration, synovitis and osteophyte hyperplasia, among which cartilage degeneration is the main characteristic (14). Therefore, the alleviation of cartilage degeneration may be key to the treatment of OA. Normal articular cartilage comprises chondrocytes and extracellular matrix, which are in a state of dynamic equilibrium. Alterations in chondrocyte proliferation, apoptosis and other physiological functions may cause dynamic balance disorders, which affect cartilage functional status (15). Therefore, chondrocytes may represent a valuable focus of investigation in OA research. It has been reported that levels of nitrite, a stable end product of nitric oxide (NO) metabolism, are elevated in serum and synovial fluid samples of OA (16). In addition, synovial cells and cartilage cells in OA produce large amounts of NO (17). The negative effects of NO include enhancement of matrix metalloproteinase activity, a reduction in interleukin-1 receptor antagonist synthesis and the promotion of apoptosis, which are closely associated with the occurrence and development of OA (18-20). Sodium nitroprusside (SNP), which is a widely used NO donor, is extremely unstable and produces NO when added to chondrocyte culture fluids $(21,22)$. To the best of our knowledge, no previous studies have used SNP to generate animal models of OA; however, SNP-induced chondrocytes are common in vitro models of OA (23-25). The present study aimed to determine whether EA may serve a therapeutic role in OA by inhibiting SNP-induced chondrocyte apoptosis.

\section{Materials and methods}

Animals. Chondrocytes were obtained from 4-week-old male Sprague Dawley rats $(n=30$; weight, $70 \pm 10$ g) purchased from Shanghai SLAC Laboratory Animal Co., Ltd. (Shanghai, China; permit no. SCXK 2012-0002). The rats were raised in the Animal Experimental Center of Fujian University of Traditional Chinese Medicine (permit no. SYXK 2014-0005; Fujian, China) at a room temperature of $24 \pm 2^{\circ} \mathrm{C}$, a relative humidity of $55 \pm 5 \%$, a $12 / 12 \mathrm{~h}$ light/dark cycle and free access to food and water. The present study was approved by the Animal Care and Use Committee of Fujian University of Traditional Chinese Medicine.

Chondrocyte acquisition and culture. Articular cartilage cells were isolated and cultured as previously described (26). After the rats were euthanized, their knee joints were removed and transferred to a clean bench for further processing. Cartilage tissue from the rat knees was removed and rinsed three times with PBS (HyClone; GE Healthcare Life Sciences, Logan, UT, USA). The cartilage tissue was digested with type II collagenase (Sigma-Aldrich; Merck KGaA, Darmstadt, Germany) in an incubator (Heraeus Holding $\mathrm{GmbH}$, Hanau, Germany) at $37^{\circ} \mathrm{C}$ and $5 \% \mathrm{CO}_{2}$ after mincing. After $90 \mathrm{~min}$, the supernatant was collected and centrifuged at $503.1 \mathrm{x}$ g for $3 \mathrm{~min}$ to achieve cell precipitation. The cell pellet was suspended in $4 \mathrm{ml}$ Dulbecco's modified Eagle's medium (DMEM) containing 10\% fetal bovine serum (both from HyClone; GE Healthcare Life Sciences).
The cell suspension was transferred to a $25-\mathrm{mm}^{2}$ flask, and was then cultured in the incubator at $37^{\circ} \mathrm{C}$ and $5 \% \mathrm{CO}_{2}$. The cartilage tissues were digested four times repeatedly. The culture medium was replenished every 2 days. Passages were performed when chondrocytes had grown to $90 \%$ confluence. Second-generation chondrocytes were employed for subsequent experiments.

Chondrocyte observation and identification. Chondrocyte morphology on different culture days and of different generations was observed under an inverted phase-contrast microscope (Leica Microsystems, Inc., Wetzlar, Germany) and images were captured. Second-generation chondrocytes are often selected for experimentation (27); therefore, type II collagen immunohistochemistry was applied to identify passage 2 chondrocytes. A total of $5 \times 10^{4}$ second-generation chondrocytes per well were implanted onto a sterile round coverglass in a 6-well plate. Chondrocytes in the 6-well plate ( $2 \mathrm{ml} \mathrm{medium/well)} \mathrm{were} \mathrm{incubated} \mathrm{for} 48 \mathrm{~h}$ and were then randomly divided into two groups. The positive group was treated with $100 \mu 1$ rabbit polyclonal antibody against collagen II (dilution 1:200; cat. no. ab34712; Abcam, Cambridge, UK), whereas the negative group was treated with $100 \mu \mathrm{l}$ PBS. Both groups were incubated overnight at $4^{\circ} \mathrm{C}$. After incubation, the two groups of chondrocytes were treated with a secondary antibody (cat. no. KIT-9707; MXB Biotechnologies, Inc., Fujian, China) at $37^{\circ} \mathrm{C}$ for $1 \mathrm{~h}$, in accordance with the manufacturer's instructions; color was developed using a DAB kit (cat. no. DAB-0031; MXB Biotechnologies, Inc.); and the cells were stained with hematoxylin (Sigma-Aldrich; Merck $\mathrm{KGaA}$ ) for $1 \mathrm{~min}$. The staining of the two groups of cells was observed and compared under a phase-contrast microscope (Leica Microsystems, Inc.).

Experimental grouping. A cell suspension $\left(1 \times 10^{5} / \mathrm{ml}\right)$ was seeded in 6-well plates $(2 \mathrm{ml} /$ well). After $72 \mathrm{~h}$, the cells were randomly divided into the following groups: i) The control group without treatment, ii) the $1 \mathrm{mM}$ SNP-treated group, iii) the group treated with $1 \mathrm{mM} \mathrm{SNP}$ and EA for 30 min every $8 \mathrm{~h}$ (electrical stimulator was obtained from Suzhou Medical Appliance Factory, Suzhou, China), and iv) the group treated with $1 \mathrm{mM} \mathrm{SNP}$ and EA for $60 \mathrm{~min}$ every $8 \mathrm{~h}$. The intervention time for all groups was $24 \mathrm{~h}$. EA intervention on chondrocytes was conducted as described previously (11).

DAPI staining. After treatment, the chondrocyte morphology in each group was observed under a microscope, and the nuclear alterations in each group were observed using DAPI staining. Initially, chondrocytes were fixed in $1 \mathrm{ml} 4 \%$ neutral paraformaldehyde (HyClone; GE Healthcare Life Sciences) at $4^{\circ} \mathrm{C}$ for $30 \mathrm{~min}$. After washing with PBS three times, the chondrocytes were stained with $500 \mu \mathrm{l}$ DAPI $(5 \mu \mathrm{g} / \mathrm{ml}$; cat. no. D1306; Thermo Fisher Scientific, Inc.) for $5 \mathrm{~min}$ in the dark. After staining, the nuclear alterations were observed and images were captured under a fluorescence microscope (Leica Microsystems, Inc.).

Annexin V-fluorescein isothiocyanate (FITC)/propidium iodide (PI) staining and flow cytometry. After treatment, chondrocytes from each group were digested with EDTA-free 
trypsin (Gibco; Thermo Fisher Scientific, Inc.) and collected in separate $15-\mathrm{ml}$ centrifuge tubes, in accordance with the instructions of the apoptosis detection kit (cat. no. KGA108; Nanjing KeyGen Biotech Co. Ltd. Nanjing, China). A total of $5 \times 10^{5}$ chondrocytes were collected in flow tubes and suspended in $500 \mu \mathrm{l}$ binding buffer. Subsequently, $5 \mu \mathrm{l}$ Annexin V-FITC and $5 \mu \mathrm{l}$ PI were added to the chondrocyte suspension and the cells were incubated in the dark for $15 \mathrm{~min}$ at room temperature. The chondrocyte apoptotic rate was measured using a fluorescence-activated cell sorting (FACS) machine (BD FACSCalibur $^{\mathrm{TM}}$; BD Biosciences, San Jose, CA, USA).

JC-1 staining and flow cytometry. After treatment, chondrocytes were digested with EDTA-free trypsin and were collected in separate $15-\mathrm{ml}$ centrifuge tubes. A total of $5 \times 10^{5}$ chondrocytes in flow tubes were then suspended in $500 \mu \mathrm{l}$ PBS containing JC-1 (cat. no. 420200; Calbiochem; Merck $\mathrm{KGaA}$ ) at $10 \mu \mathrm{g} / \mathrm{ml}$. The mixture was placed in a cell incubator at $37^{\circ} \mathrm{C}$ and $5 \% \mathrm{CO}_{2}$ for $15 \mathrm{~min}$. Alterations in mitochondrial membrane potential were then detected using the $\mathrm{BD}$ FACSCalibur $^{\mathrm{TM}}$ (BD Biosciences).

Reverse transcription-quantitative polymerase chain reaction $(R T-q P C R)$. After treatment, TRIzol ${ }^{\circledR}$ reagent (Invitrogen; Thermo Fisher Scientific, Inc.) was applied to extract total RNA. The PrimeScript ${ }^{\mathrm{TM}}$ RT reagent kit (cat. no. RR0047A; Takara Bio, Inc., Otsu, Japan) was used to reverse transcribe RNA $(1 \mu \mathrm{g})$ into cDNA in the PCR amplification instrument according to the manufacturer's protocol. The primer sequences were as follows: B-cell lymphoma 2 (Bcl-2), forward 5'-TCCAGGCATCAGGTTAGTC-3', reverse 5'-GGT CAGTGTCCAGGTAGG-3'; Bcl-2-associated X protein (Bax), forward 5'-TGTCAGTCCTGGCAGTCAAC-3', reverse 5'-GGCTCAGTAGTAGGCGATGG-3'; caspase-9, forward 5'-AATGGATGTGGTGCTGTC-3', reverse 5'-AACTGTATA GGAAGGCTGAG-3'; caspase-3, forward 5'-TACAGGAAC AGACCATAATACC-3', reverse 5'-AGACCAGTGCTCACA AGG-3'; and $\beta$-actin, forward 5'-ACCACTGGCATTGTG ATG GA-3' and reverse 5'-CGCTCGGTCAGGATCTTC T-3' (Sangon Biotech Co., Ltd., Shanghai, China). The qPCR SYBR Green Master Mix (cat. no. Q111-02; Vazyme Biotech Co., Ltd., Nanjing, China) was used to detect the respective mRNA expression levels. The total reaction volume was $20 \mu 1$, including $10 \mu 1 \mathrm{qPCR}$ SYBR Green Master Mix, $8 \mu 1$ sterilized distilled water, $1 \mu \mathrm{l}$ cDNA, $0.5 \mu 1$ forward primer and $0.5 \mu \mathrm{l}$ reverse primer. RT-qPCR was conducted using the 7500 Fast Real-Time PCR system (Applied Biosystems; Thermo Fisher Scientific, Inc.) with the following reaction conditions: Stage 1, pre-denaturation at $95^{\circ} \mathrm{C}$ for $3 \mathrm{~min}$; stage 2,40 cycles at $95^{\circ} \mathrm{C}$ for $10 \mathrm{sec}$ and $60^{\circ} \mathrm{C}$ for $30 \mathrm{sec}$; stage 3 , dissolution curve at $95^{\circ} \mathrm{C}$ for $15 \mathrm{sec}, 60^{\circ} \mathrm{C}$ for $60 \mathrm{sec}$ and $95^{\circ} \mathrm{C}$ for $15 \mathrm{sec}$. The $2^{-\Delta \Delta \mathrm{Cq}}$ method (28) was applied for data analysis.

Western blot analysis. Chondrocytes from the four groups were lysed on ice for $30 \mathrm{~min}$ with radioimmunoprecipitation assay lysis buffer and phenylmethanesulfonyl fluoride (both from Beyotime Institute of Biotechnology, Shanghai, China), in order to extract proteins. A bicinchoninic acid kit (cat. no. P0010; Beyotime Institute of Biotechnology) was applied to determine protein concentrations in the
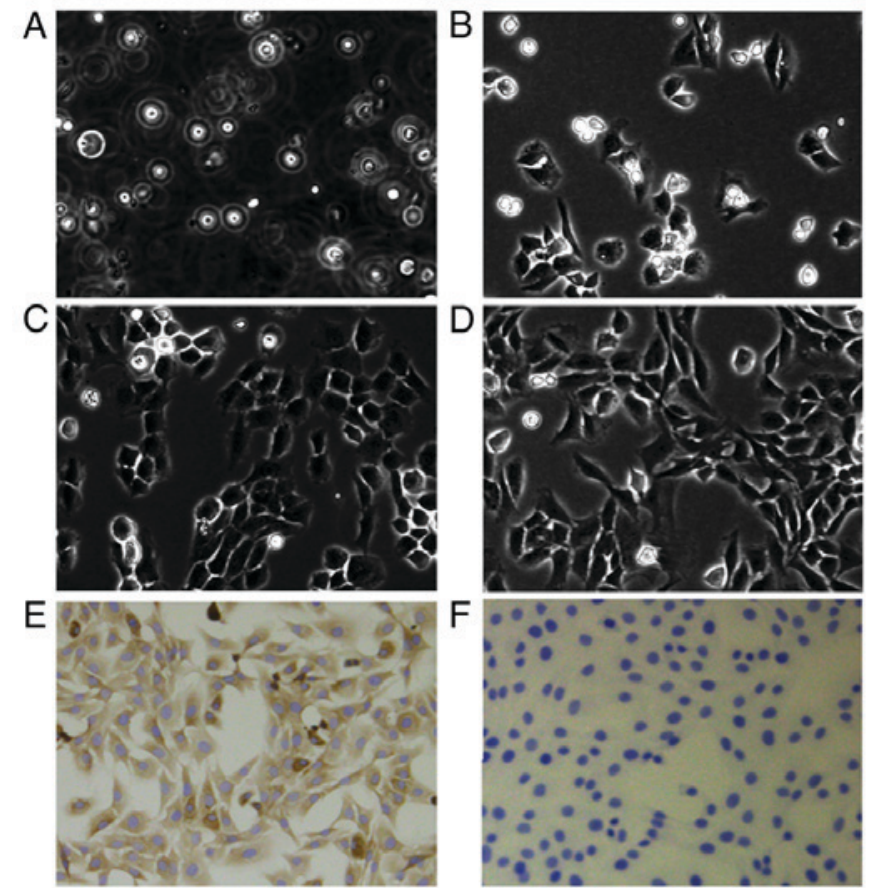

Figure 1. Observation and identification of chondrocytes (magnification, x100). (A-D) Cartilage cells were observed under an inverted phase contrast microscope. (A) Newly extracted cartilage cells. (B) Newly isolated cells cultured for 2 days. (C) Chondrocytes grown for 2 days after one passage. (D) Chondrocytes grown for 2 days after two passages. (E and F) Chondrocytes were identified by type II collagen immunohistochemistry. (E) Cytoplasm of positive second-generation chondrocytes was stained brown. (F) Negative control cells were not stained.

different groups. Protein samples $(20 \mu \mathrm{g})$ were separated by $12 \%$ SDS-PAGE and were transferred to polyvinylidene fluoride membranes. Blocking buffer (Beyotime Institute of Biotechnology) was used to block the membranes at room temperature for $1 \mathrm{~h}$. After $1 \mathrm{~h}$, the membranes were incubated with antibodies against Bax (1:5,000 dilution; cat. no. ab32503; Abcam), Bcl-2 (1:1,000 dilution; cat. no. 2870; Cell Signaling Technology, Inc., Danvers, MA, USA) and $\beta$-actin (1:1,000 dilution; cat. no. 8457; Cell Signaling Technology, Inc.) at $4^{\circ} \mathrm{C}$ overnight, followed by incubation with secondary antibodies (1:20,000 dilution; cat. no. AP132P; Merck KGaA) at room temperature for $1 \mathrm{~h}$. Finally, protein expression semi-quantified using the ChemiDoc $^{\text {TM }}$ XRS $^{+}$system (Bio-Rad Laboratories, Inc., Hercules, CA, USA) with BeyoECL Plus (Beyotime Institute of Biotechnology).

Colorimetric assays. Two colorimetric kits (cat. nos. ab65608 and ab39401; Abcam) were applied to detect caspase-9 and caspase-3 activity. The experimental procedure was conducted according to the manufacturer's protocol. After the chondrocytes were completely lysed, the mixture was centrifuged at $10,000 \mathrm{x} \mathrm{g}$ for $1 \mathrm{~min}$ at $4^{\circ} \mathrm{C}$ to obtain total cellular protein. The total reaction volume for this experiment included $100 \mu \mathrm{g}$ protein dissolved in $50 \mu \mathrm{l}$ cell lysis buffer, substrate, $50 \mu \mathrm{l}$ reaction buffer and either $5 \mu 1$ LEHD-p-NA (for caspase-9 detection) or DEVD-p-NA (for caspase-3 detection; all reagents contained within relevant kits); this reaction mixture was mixed thoroughly in 96-well plates and then incubated 

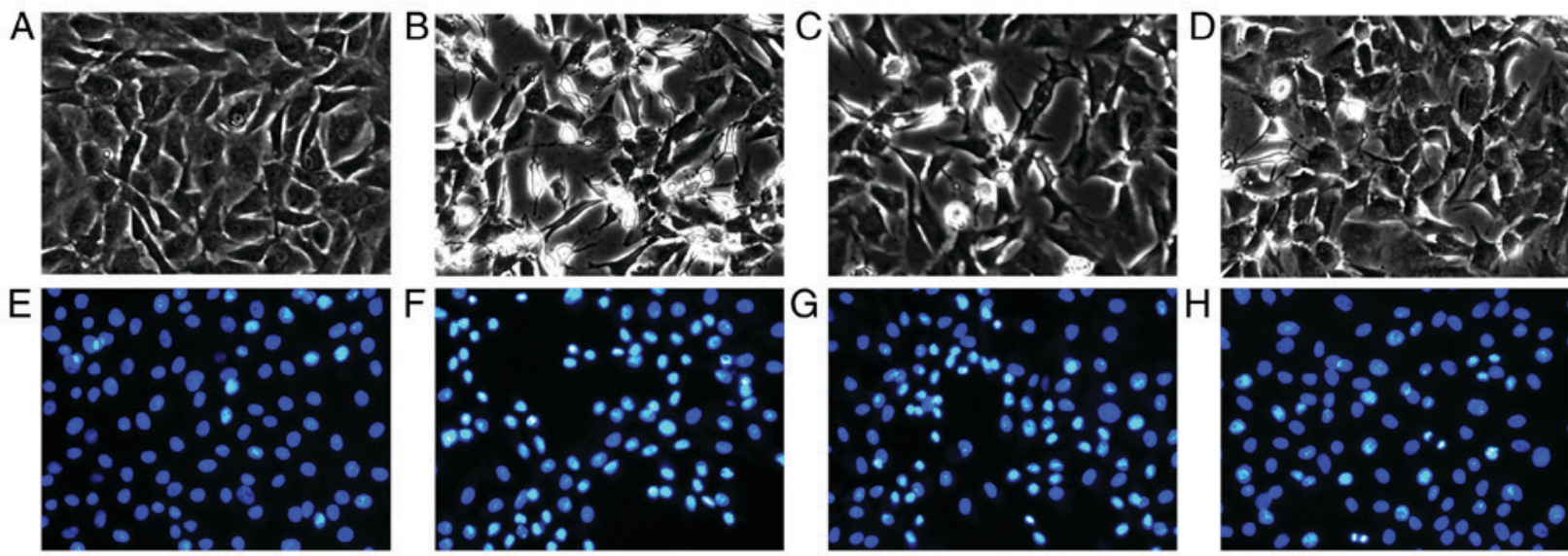

Figure 2. EA is associated with morphological alterations in apoptotic chondrocytes (magnification, x100). (A-D) Chondrocyte microstructure. (E-H) DAPI-stained chondrocytes. (A and E) Normal chondrocytes. (B and F) Cells treated with $1 \mathrm{mM} \mathrm{SNP}$ for $24 \mathrm{~h}$. (C and G) Cells treated with $1 \mathrm{mM}$ SNP and EA for 30 min every $8 \mathrm{~h}$ for $24 \mathrm{~h}$. (D and H) Cells treated with $1 \mathrm{mM} \mathrm{SNP}$ and EA for 60 min every $8 \mathrm{~h}$ for $24 \mathrm{~h}$. EA, electroacupuncture; SNP, sodium nitroprusside.

in the dark at $37^{\circ} \mathrm{C}$ for $2 \mathrm{~h}$. The optical density of the samples was measured at $405 \mathrm{~nm}$ using a microplate reader (BioTek Instruments, Inc., Winooski, VT, USA).

Immunofluorescence staining. A total of $1 \times 10^{5} / \mathrm{ml}$ chondrocytes were seeded in a laser confocal dish. After $72 \mathrm{~h}$, the cells were treated with either SNP or EA. Following this, chondrocytes were fixed with $500 \mu \mathrm{l}$ formaldehyde at $4^{\circ} \mathrm{C}$ for $30 \mathrm{~min}$ and then incubated with $500 \mu 10.5 \%$ Triton at room temperature for $10 \mathrm{~min}$ in order to increase cell membrane permeability. After blocking with PBS containing $10 \%$ goat serum (cat. no. SL038; Beijing Solarbio Science $\&$ Technology Co., Ltd., Beijing, China) and $0.5 \%$ bovine serum albumin (cat. no. A8010; Beijing Solarbio Science $\&$ Technology Co., Ltd) at room temperature for $1 \mathrm{~h}$, the chondrocytes were incubated with anti-cytochrome $c$ (Cyt-C; 1:500 dilution; cat. no. ab90529; Abcam) at $4{ }^{\circ} \mathrm{C}$ overnight. Following this, chondrocytes were incubated with a fluorescent secondary antibody (1:300 dilution; cat. no. A-11008; Thermo Fisher Scientific, Inc.) for $1 \mathrm{~h}$ at room temperature and with DAPI $(5 \mu \mathrm{g} / \mathrm{ml})$ for $5 \mathrm{~min}$ at room temperature in the dark. After three washes with PBS, the chondrocytes were observed and images were captured under a laser scanning confocal microscope (Olympus Corporation, Tokyo, Japan). ImageJ 1.8.0 (https://imagej.nih.gov/ij/download. html) was used to measure the fluorescence intensity of $\mathrm{Cyt}-\mathrm{C}$ in different images, and the level of intensity per area was used to indicate the relative expression of $\mathrm{Cyt}-\mathrm{C}$. Three visual fields from each laser confocal dish were randomly selected for data analysis.

Statistical analysis. Each experiment was independently repeated at least three times. Experimental data were processed and analyzed using SPSS 22.0 software (IBM Corp., Armonk, NY, USA). The Shapiro-Wilk test was used to determine the normality of all groups of data. If the data exhibited a normal distribution, they were analyzed with one-way analysis of variance followed by least significant difference or Games Howell post hoc tests; if not, the Kruskal-Wallis test was used and the Mann-Whitney U with Bonferroni's correction was applied as a post hoc test. $\mathrm{P}<0.05$ was considered to indicate a statistically significant difference.

\section{Results}

Morphological observation of chondrocytes isolated from the articular cartilage of rats. Cells that had just been extracted from rat articular cartilage were suspended in culture medium (Fig. 1A). After 2 days, the majority of the cells were adherent to the culture bottle and had an irregular shape (Fig. 1B). Firstand second-generation chondrocytes after 2 days of culture assumed a round or oval shape (Fig. 1C and D). Briefly, the rat joint cartilage cell morphology resembled that reported in previous studies $(26,27)$.

Detection of type II collagen in second-generation chondrocytes. Since type II collagen is mainly produced by chondrocytes, type II collagen immunohistochemistry was used to identify second-generation chondrocytes. Under an inverted phase contrast microscope, the cytoplasm of chondrocytes in the positive group was stained brown (Fig. 1E), whereas the cytoplasm of the chondrocytes in the negative group was not stained (Fig. 1F). Since second-generation articular cartilage cells exhibited the typical morphology of chondrocytes and abundant type II collagen, they were employed in subsequent experiments.

EA inhibits SNP-induced chondrocyte apoptosis, as determined by morphological observation and DAPI staining. Microscopic observation and DAPI staining were performed to investigate the effects of EA on SNP-induced apoptotic cells (Fig. 2). Chondrocyte morphology in the normal group exhibited no obvious alterations (Fig. 2A), and the nuclei were stained blue, and were round- or oval-shaped (Fig. 2E). However, in the SNP-induced group, numerous chondrocytes were observed floating in the culture medium, and adherent cells had an irregular morphology (Fig. 2B). Compared with the normal group, the nuclei of the SNP-treated group were significantly reduced in size and were bright blue (Fig. 2F). Following EA treatment, there were fewer chondrocytes 

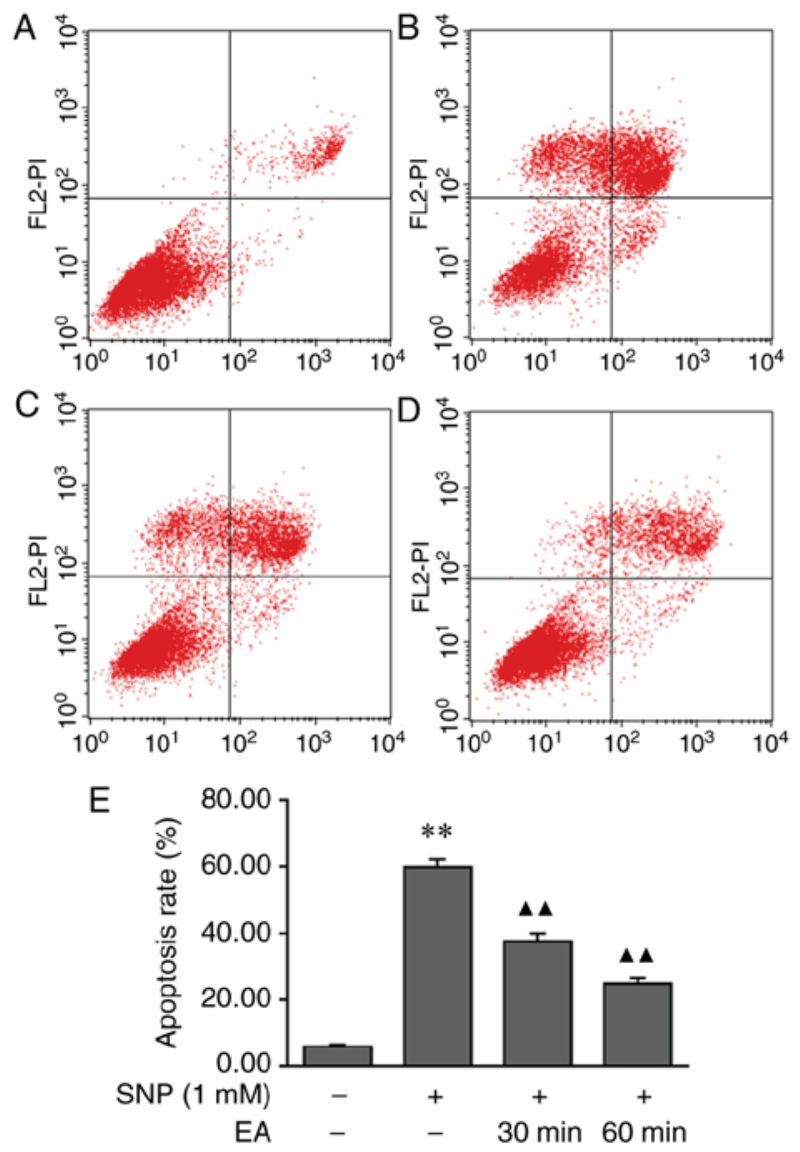

Figure 3. EA reduces the apoptotic rate of chondrocytes. (A) Normal chondrocytes, and chondrocytes treated with (B) $1 \mathrm{mM} \mathrm{SNP,(C)} 1 \mathrm{mM} \mathrm{SNP}$ and EA for $30 \mathrm{~min}$ per $8 \mathrm{~h}$, and (D) $1 \mathrm{mM} \mathrm{SNP}$ and EA for $60 \mathrm{~min}$ per $8 \mathrm{~h}$ for $24 \mathrm{~h}$ were analyzed by flow cytometry. (E) Apoptotic rates of chondrocytes treated with SNP with or without EA are summarized in the histogram. Data from three different experiments are presented as the means \pm standard deviation. ${ }^{* * *} \mathrm{P}<0.01$ vs. normal chondrocytes; ${ }^{\boldsymbol{\Delta}} \mathrm{P}<0.05$ and ${ }^{\boldsymbol{\Delta}}{ }^{\mathbf{A}} \mathrm{P}<0.01$ vs. SNP-treated chondrocytes. EA, electroacupuncture; SNP, sodium nitroprusside.

floating in the culture medium (Fig. 2C and D), and the shrinking and brightness of the nuclei were less prominent compared with in the SNP-treated group (Fig. 2G and H).

EA modulates the apoptotic rate of SNP-induced chondrocytes. The effects of EA on chondrocyte apoptosis were assessed by Annexin V-FITC/PI staining and flow cytometry. The results demonstrated that the apoptotic rate of SNP-induced chondrocytes was significantly higher compared with that of normal chondrocytes $(\mathrm{P}<0.001)$. Conversely, the apoptotic rate of chondrocytes following EA treatment was lower compared with that of SNP-induced chondrocytes $(\mathrm{P}<0.001)$. These findings indicated that EA reduced the apoptotic rate of chondrocytes (Fig. 3).

EA decelerates the reduction in mitochondrial membrane potential in SNP-stimulated apoptotic chondrocytes. JC-1 single-staining flow cytometry was performed to determine the effects of EA on the mitochondrial membrane potential of apoptotic chondrocytes. The mitochondrial membrane potential was significantly decreased following treatment of chondrocytes with $1 \mathrm{mM}$ SNP for $24 \mathrm{~h}(\mathrm{P}=0.001)$. Compared with in the SNP-induced group, the decrease in mitochondrial
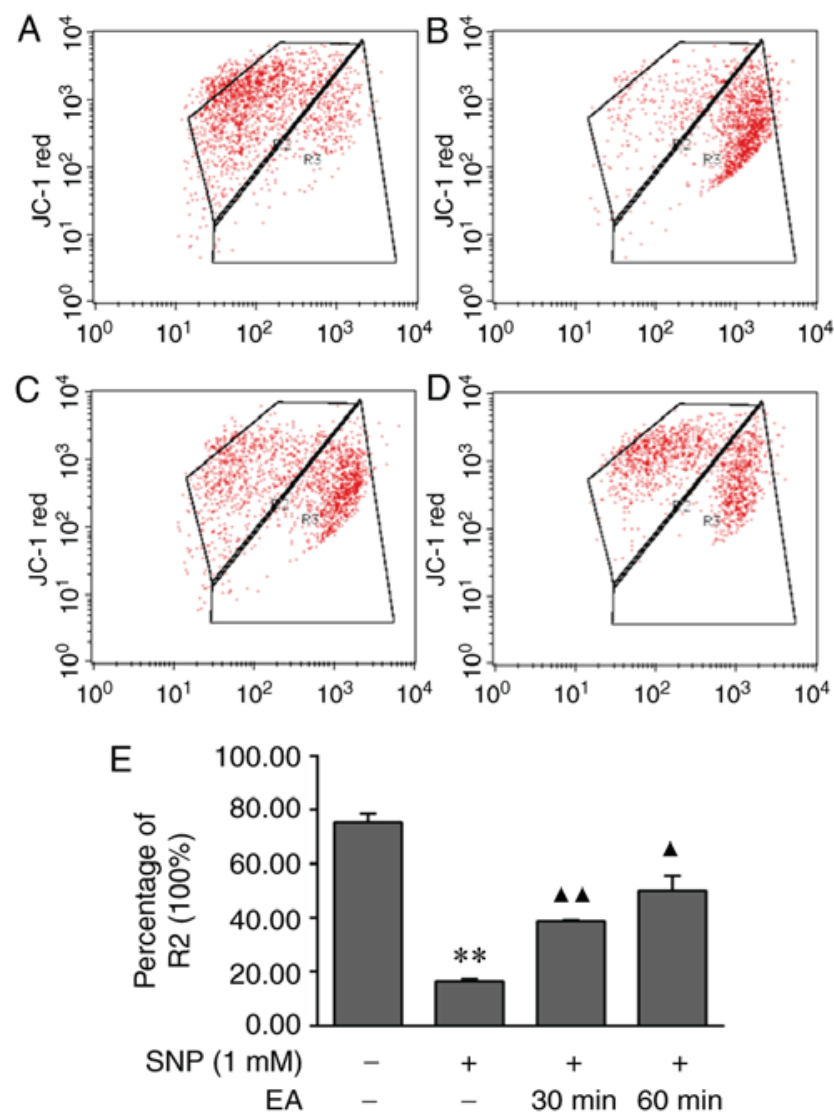

Figure 4. EA decelerates the mitochondrial membrane potential of apoptotic chondrocytes. (A) Normal chondrocytes, and chondrocytes treated with (B) $1 \mathrm{mM} \mathrm{SNP}$, (C) $1 \mathrm{mM} \mathrm{SNP}$ and EA for 30 min every $8 \mathrm{~h}$, and (D) $1 \mathrm{mM}$ SNP and EA for 60 min every $8 \mathrm{~h}$ for $24 \mathrm{~h}$ were analyzed by JC-1 staining and flow cytometry. (E) R2 percentage of cartilage cells treated with SNP with or without EA is summarized in the histogram. Data from three different experiments are presented as the means \pm standard deviation. ${ }^{* *} \mathrm{P}<0.01 \mathrm{vs}$ normal chondrocytes; ${ }^{\mathbf{\Delta}} \mathrm{P}<0.05$ and ${ }^{\boldsymbol{\Delta} \mathbf{\Delta}} \mathrm{P}<0.01$ vs. SNP-treated chondrocytes. EA, electroacupuncture; SNP, sodium nitroprusside.

membrane potential was significantly slower in the EA treatment groups $(\mathrm{P}<0.001, \mathrm{P}=0.018)$. The results demonstrated that EA increased the mitochondrial membrane potential of apoptotic chondrocytes (Fig. 4).

EA regulates gene and protein expression in chondrocytes. The aforementioned results indicated that EA may inhibit $1 \mathrm{mM}$ SNP-induced chondrocyte apoptosis. To further elucidate the mechanism of action of EA at the molecular level, the expression levels of Bcl-2, Bax, Cyt-C, caspase-9 and caspase-3 were assessed. RT-qPCR analysis (Fig. 5) indicated that the mRNA expression levels of Bcl-2 in SNP-induced chondrocytes were reduced compared with in normal chondrocytes $(\mathrm{P}=0.001)$. Furthermore, the mRNA expression levels of Bax $(\mathrm{P}=0.003)$, caspase-9 $(\mathrm{P}<0.001)$ and caspase-3 $(\mathrm{P}<0.001)$ were significantly increased in SNP-induced chondrocytes compared with in normal chondrocytes. Conversely, compared with in SNP-induced chondrocytes, EA treatment for 30 and $60 \mathrm{~min}$ promoted Bcl-2 mRNA expression $(\mathrm{P}=0.159, \mathrm{P}=0.032)$, and reduced $\operatorname{Bax}(\mathrm{P}=0.028, \mathrm{P}=0.018)$, caspase-9 $(\mathrm{P}=0.005, \mathrm{P}=0.002)$ and caspase $-3(\mathrm{P}=0.029, \mathrm{P}=0.002) \mathrm{mRNA}$ expression.

Western blotting was conducted to detect the protein expression levels of Bcl-2 and Bax (Fig. 6A-C). In addition, 
A

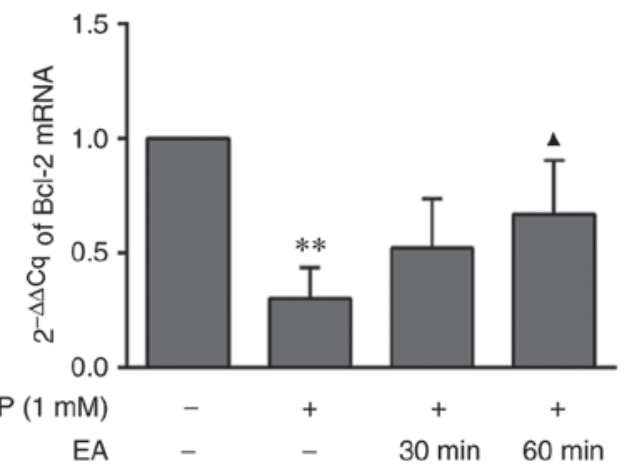

C

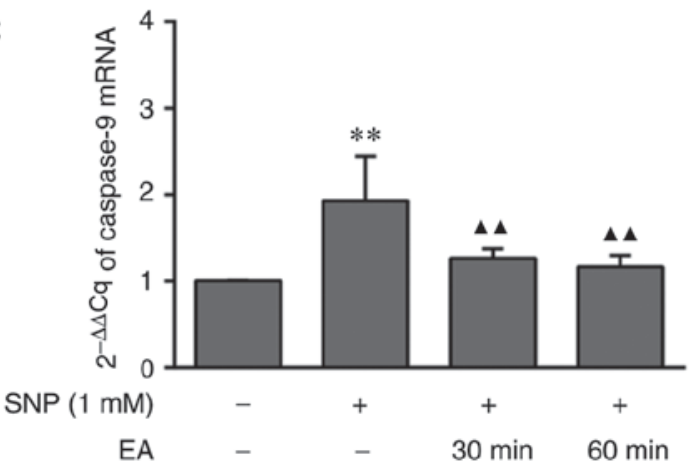

B

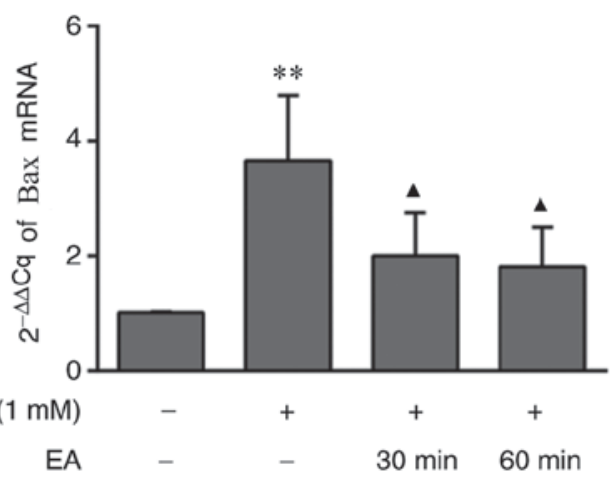

D

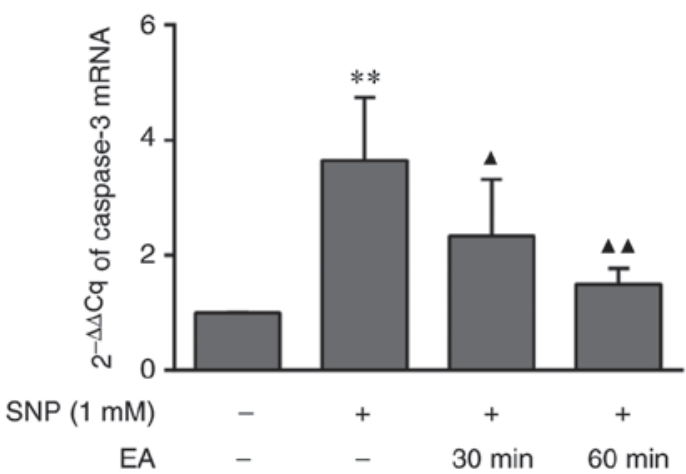

Figure 5. Expression of genes in chondrocytes treated with SNP and/or EA. (A) Bcl-2, (B) Bax, (C) caspase-3 and (D) caspase-9 mRNA expression levels were detected by reverse transcription-quantitative polymerase chain reaction. Data from three different experiments are presented as the means \pm standard deviation. ${ }^{* *} \mathrm{P}<0.01$ vs. normal chondrocytes; ${ }^{\boldsymbol{\Delta}} \mathrm{P}<0.05$ and ${ }^{\boldsymbol{\Delta}}{ }^{\boldsymbol{\Delta}} \mathrm{P}<0.01$ vs. SNP-treated chondrocytes. Bax, Bcl-2-associated $\mathrm{X}$ protein; Bcl-2, B-cell lymphoma 2; EA, electroacupuncture; SNP, sodium nitroprusside.

caspase-9 and caspase-3 activities were measured using colorimetric assays (Fig. 6D and E), and immunofluorescence staining was used to detect the expression of Cyt-C (Fig. 6F and G). SNP inhibited Bcl-2 protein expression $(\mathrm{P}=0.001)$, promoted Bax $(\mathrm{P}=0.007)$ and $\mathrm{Cyt}-\mathrm{C}(\mathrm{P}<0.001)$ protein expression, and enhanced caspase-3 $(\mathrm{P}=0.005)$ and caspase-9 $(\mathrm{P}=0.001)$ activities. Conversely, EA treatment for 30 and $60 \mathrm{~min}$ increased $\mathrm{Bcl}-2$ protein expression $(\mathrm{P}=0.083, \mathrm{P}=0.012)$, downregulated $\mathrm{Bax} \quad(\mathrm{P}=0.047$, $\mathrm{P}=0.021)$ and $\mathrm{Cyt}-\mathrm{C}$ (both $\mathrm{P}<0.001)$ protein expression, and reduced caspase-9 (both $\mathrm{P}=0.001)$ and caspase- $3(\mathrm{P}=0.049$, $\mathrm{P}=0.009)$ activities.

\section{Discussion}

The present study demonstrated that EA suppressed SNP-mediated chondrocyte apoptosis, and exerted an inhibitory effect on apoptosis through modulation of the mitochondrial pathway.

Chondrocytes, which are the only type of cell present in mature cartilage, synthesize and secrete matrix components and fibers, thus maintaining cartilage tissue structure and function (29). Cartilage degeneration is the most important pathological manifestation of OA, and chondrocyte apoptosis is closely associated with cartilage degeneration (30). Therefore, it has been hypothesized that effective inhibition of chondrocyte apoptosis is key to the treatment of OA $(31,32)$. In previous studies, cartilage histomorphological examination revealed that EA treatment significantly inhibits cartilage degeneration in an ovariectomized rabbit OA model and an anterior cruciate ligament transection rabbit OA model $(33,34)$. In addition, a previous TUNEL assay demonstrated that EA reduces the rate of chondrocyte apoptosis in cartilage tissue (35), which was similar to the present findings. However, to the best of our knowledge, the mechanism through which EA inhibits the apoptosis of chondrocytes has not been extensively investigated to date.

SNP is a nitrosylated sodium ferricyanide dihydrate, which readily releases $\mathrm{NO}$, as it contains an extremely unstable nitroso group. NO, which is an important mitochondrial apoptosis-inducing factor, may cause chondrocyte apoptosis (21). Based on previous experiments, $1 \mathrm{mM} \mathrm{SNP}$ was used for $24 \mathrm{~h}$ to induce chondrocyte apoptosis. After treatment, it was revealed that EA improved morphological alterations in apoptotic chondrocytes. DAPI, a fluorescent DNA-binding dye (36), is widely used to evaluate apoptosis. DAPI staining revealed that the nuclei of chondrocytes treated with SNP and EA exhibited less shrinkage, reduced brightness and higher nuclear density compared with SNP-treated chondrocytes. Furthermore, Annexin V-FITC/PI staining was used to assess overall chondrocyte apoptosis. When cells undergo apoptosis, phosphatidylserine (PS), originally located inside the lipid bilayer, is relocated to the outer surface of the bilayer (37). Annexin-V, which binds strongly to PS, may be used to label early apoptotic cells (38). PI is a nucleic acid dye that does not penetrate the intact cell membrane, but permeates the cell membrane of late apoptotic and dead cells (39). Annexin V-FITC/PI staining is a classic experimental method for the detection of apoptosis. 

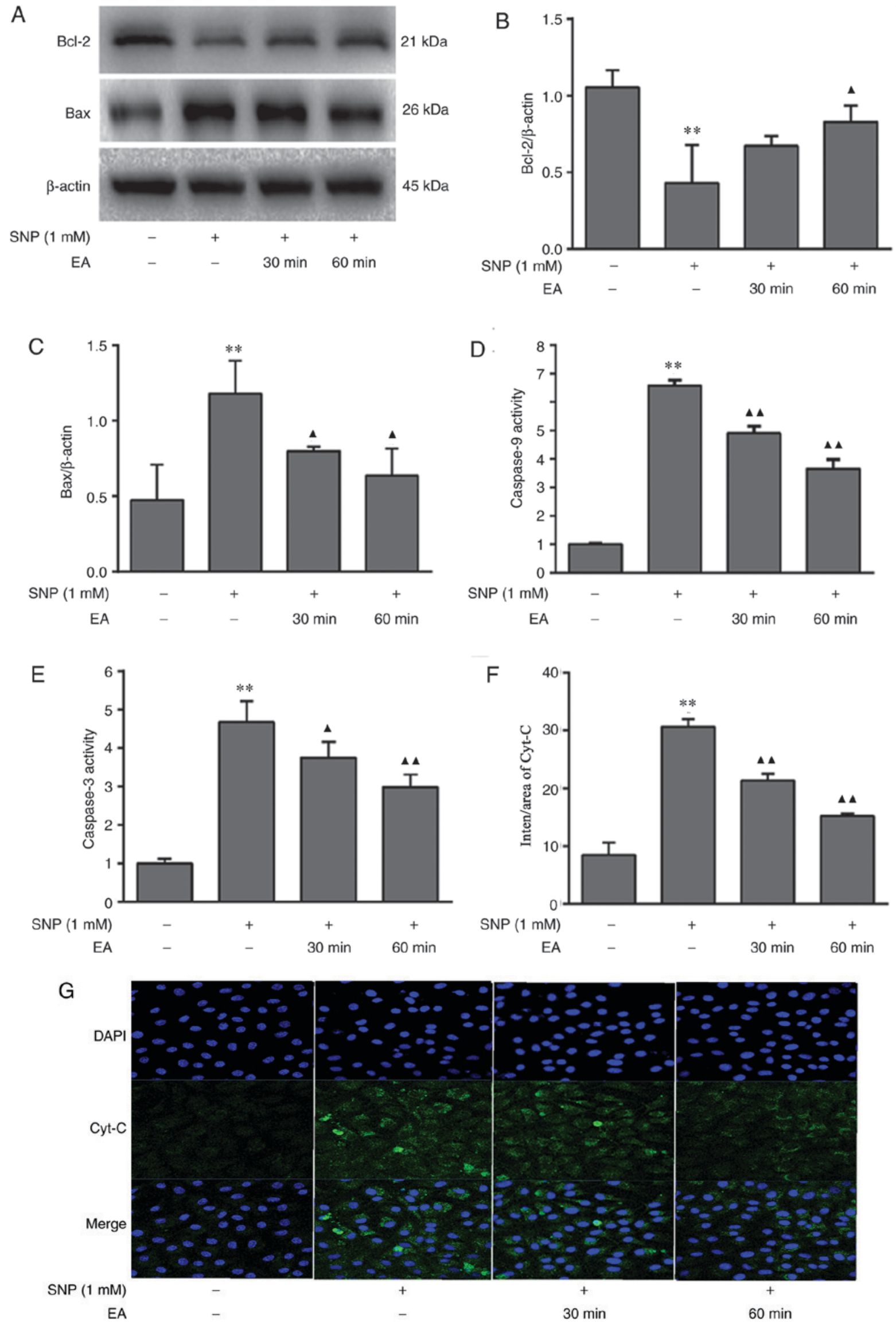

Figure 6. Expression of proteins in chondrocytes treated with SNP and/or EA. (A-C) Bcl-2 and Bax protein expression was measured and results were semi-quantified. (D and E) Colorimetric assays were performed to detect caspase-9 and caspase-3 activities. (F and G) Cyt-C expression was evaluated by laser scanning confocal microscopy (magnification, x400) and the results were semi-quantified. Data from three different experiments are presented as the means \pm standard deviation. ${ }^{* *} \mathrm{P}<0.01$ vs. normal chondrocytes; ${ }^{\mathbf{\Lambda}} \mathrm{P}<0.05$ and ${ }^{\boldsymbol{\Delta} \mathbf{\Delta}} \mathrm{P}<0.01$ vs. SNP-treated chondrocytes. Bax, Bcl-2-associated $\mathrm{X}$ protein; Bcl-2, B-cell lymphoma 2; Cyt-C, cytochrome $c$; EA, electroacupuncture; OD, optical density; SNP, sodium nitroprusside. 
In this study, EA reduced the rate of chondrocyte apoptosis, as demonstrated by the results of Annexin V-FITC/PI staining.

Having confirmed that EA inhibited SNP-induced chondrocyte apoptosis, further analysis was conducted. Since SNP has been reported to induce mitochondrial apoptosis (40), alterations in mitochondrial membrane potential, and associated gene and protein expression levels, were investigated. Mitochondrial membrane potential is often detected using JC-1 (41). In the JC-1 assay, mitochondrial membrane potential decline, which reflects the onset of apoptosis, is indicated by the change in fluorescence from red to green. A decrease in mitochondrial membrane potential leads to increased membrane permeability, and mitochondrial membrane permeability may be regulated by the Bcl-2 family (42). The Bcl-2 family promotes and inhibits cell apoptosis through modulating mitochondrial outer membrane permeabilization (43). Bcl-2 can suppress apoptosis by inhibiting the increase in mitochondrial permeability (44). Conversely, Bax, another Bcl-2 family member, accelerates apoptosis. The Bax protein increases the permeability of the mitochondrial membrane by forming activated oligomers, promoting Cyt-C release and ultimately inducing apoptosis (45). When cells are stimulated by NO, mitochondrial membrane permeability increases, which leads to $\mathrm{Cyt}-\mathrm{C}$ release into the cytoplasm, triggering the caspase cascade, namely sequential activation of caspase-9 and caspase-3, leading to cell apoptosis (46). In the present study, EA reduced the extent of the mitochondrial membrane potential decline, and regulated the expression levels of Bcl-2, Bax, Cyt-C, caspase-9 and caspase-3.

In conclusion, EA inhibited SNP-induced chondrocyte apoptosis through regulating the mitochondrial pathway, which is likely the mechanism of action of EA in OA treatment. However, there were certain limitations to the present study. The experiments were only conducted in vitro, and the results have yet to be further validated in vivo. In addition, the experiments regarding the inhibitory effects of EA on apoptosis focused only on the mitochondrial pathway; therefore, more in-depth research is required. Further in vitro and in vivo experiments must be conducted to fully elucidate the mechanism underlying the action of EA in the treatment of OA.

\section{Acknowledgements}

Not applicable.

\section{Funding}

The present study was funded by the National Natural Science Foundation of China (grant no. 81373719) and the Fujian Provincial Development and Reform Commission (grant no. 2014-514).

\section{Availability of data and materials}

The datasets used during the present study are available from the corresponding author on reasonable request.

\section{Authors' contributions}

GW, XL and MW conceived and designed the study. JL, JC, $\mathrm{CF}$ and $\mathrm{XH}$ performed the experiments. LL performed the data analysis. JL and LL wrote the manuscript. XL and GW reviewed and edited the manuscript. All authors approved the final version of the manuscript.

\section{Ethics approval and consent to participate}

The present study was approved by the Animal Care and Use Committee of Fujian University of Traditional Chinese Medicine.

\section{Patient consent for publication}

Not applicable.

\section{Competing interests}

The authors declare that they have no competing interests.

\section{References}

1. Chu CR, Millis MB and Olson SA: Osteoarthritis: From palliation to prevention: AOA critical issues. J Bone Joint Surg Am 96: e130, 2014.

2. Cross M, Smith E, Hoy D, Nolte S, Ackerman I, Fransen M, Bridqett L, Williams S, Guillemin F, Hill CL, et al: The global burden of hip and knee osteoarthritis: Estimates from the global burden of disease 2010 study. Ann Rheum Dis 73: 1323-1330, 2014.

3. Prieto-Alhambra D, Judqe A, Javaid MK, Cooper C, Diez-Perez A and Arden NK: Incidence and risk factors for clinically diagnosed knee, hip and hand osteoarthritis: Influences of age, gender and osteoarthritis affecting other joints. Ann Rheum Dis 73: 1659-1664, 2014

4. Yu D, Jordan KP, Bedson J, Enqlund M, Blyth F, Turkiewicz A, Prieto-Alhambra D and Peat G: Population trends in the incidence and initial management of osteoarthritis: Age-period-cohort analysis of the clinical practice research datalink, 1992-2013. Rheumatology (Oxford) 56: 1902-1917, 2017.

5. Lanas A, Tornero J and Zamarano JL: Assessment of gastrointestinal and cardiovascular risk in patients with osteoarthritis who require NSAIDs: The LOGICA study. Ann Rheum Dis 69: 1453-1458, 2010

6. Arrich J, Piribauer F, Mad P, Schmid D, Klaushofer K and Mullner M: Intra-articular hyaluronic acid for the treatment of osteoarthritis of the knee: Systematic review and meta-analysis. CMAJ 172: 1039-1043, 2005.

7. Gunaratne R, Pratt DN, Banda J, Fick DP, Khan RJK and Robertson BW: Patient dissatisfaction following total knee arthroplasty: A systematic review of the literature. J Arthroplasty 32: 3854-3860, 2017

8. Qi L, Tanq Y, You Y, Qin F, Zhai L, Peng H and Nie R: Comparing the effectiveness of electroacupuncture with different grades of knee osteoarthritis: A prospective study. Cell Physiol Biochem 39: 2331-2340, 2016.

9. Shim JW, Junq JY and Kim SS: Effects of electroacupuncture for knee osteoarthritis: A systematic review and meta-analysis. Evid Based Complement Alternat Med 2016: 3485875, 2016.

10. Chen N, Wanq J, Mucelli A, Zhang X and Wanq C: Electro-acupuncture is benedicial for knee osteoarthritis: The evidence from meta-analysis of randomized controlled trials. Am J Chin Med 45: 965-985, 2017.

11. Wu G, Peng J, Wu M, Li Y, Huang Y, Lin R, Cai Q and Liu X: Experimental study of low-frequency electroacupunctureinduced differentiation of bone marrow mesenchymal stem cells into chondrocytes. Int J Mol Med 27: 79-86, 2011.

12. Huang Y, Wu G, Fan H, Ye J and Liu X: Electroacupuncture promotes chondrocyte proliferation via accelerated G1/S transition in the cell cycle. Int J Mol Med 31: 1443-1448, 2013.

13. Chen H, Shao X, Li L, Zheng C, Xu X, Hong X, Li X and Wu M: Electroacupuncture serum inhibits TNF- $\alpha$-mediated chondrocytes inflammation via the Ras-Raf-MEK1/2-ERK1/2 signaling pathway. Mol Med Rep 16: 5807-5814, 2017.

14. Sulzbacher L: Osteoarthritis: Histology and pathogenesis. Wien Med Wochenschr 163: 212-219, 2013. 
15. Hemanth A and Anja N: Role of chondrocytes in cartilage formation, progression of osteoarthritis and cartilage regeneration. J Dev Biol 3: 177-192, 2015.

16. Jang D and Murrell GA: Nitric oxide in arthritis. Free Radic Biol Med 24: 1511-1519, 1998

17. Hayashi T, Abe E, Yamate T and Jasin HE: Nitric oxide production by superficial and deep articular chondrocytes. Arthritis Rheum 40: 261-269, 1997.

18. Hashimoto S, Takahashi K, Amiel D, Coutts RD and Lotz M: Chondrocyte apoptosis and nitric oxide production during experimentally induced osteoarthritis. Arthritis Rheum 41: 1266-1274, 1998

19. Abramson SB: Nitric oxide in inflammation and pain associated with osteoarthritis. Arthritis Res Acta 10 (Suppl 2): S2, 2008.

20. Abramson SB: Osteoarthritis and nitric oxide. Osteoarthritis Cartilage 16 (Suppl 2): S15-S20, 2008.

21. Blanco FJ, Ochs RL, Schwarz H and Lotz M: Chondrocyte apoptosis induced by notric oxide. Am J Pathol 146: 75-85, 1995.

22. Kim HA, Lee KB and Bae S: The mechanism of low-concentration sodium nitroprusside-mediated protection of chondrocytes death. Arthritis Res Ther 7: R526-R535, 2005.

23. Notoya K, Jovanovic DV, Reboul P, Martel-Pelletier J, Mineau F and Pelletier JP: The induction of cell death in human osteoarthritis chondrocytes by nitric oxide is related to the production of prostaglandin E2 via the induction of cyclooxygenase-2. J Immunol 165: 3402-3410, 2000.

24. Kühn K and Lotz M: Mechanisms of sodium nitroprusside-induced death in human chondrocytes. Rheumatol Int 23 241-247, 2003

25. Todd Allen R, Robertson CM, Harwood FL, Sasho T, Williams SK, Pomerleau AC and Amiel D: Characterization of mature vs aged rabbit articular cartilage: Analysis of cell density, apoptosis-related gene expression and mechanisms controlling chondrocyte apoptosis. Osteoarthritis Cartilage 12: 917-923, 2004.

26. Li X, Du M, Liu X, Chen W, Wu M, lin J and Wu G: Millimeter wave treatment promotes chondrocyte proliferation by upregulating the expression of cyclin-dependent kinase 2 and cyclin A. Int J Mol Med 26: 77-84, 2010.

27. Lin P, Weng X, Liu F, Ma Y, Chen H, Shao X, Zheng W, Liu X Ye $\mathrm{H}$ and Li X: Bushen Zhuangjin decoction inhibits TM-induced chondrocytes apoptosis mediated by endoplasmic reticulum stress. Int J Mol Med 36: 1519-1528, 2015.

28. Livak KJ and Schmittgen TD: Analysis of relative gene expression data using real-time quantitative PCR and the 2(-Delta Delta C(T)) method. Methods 25: 402-408, 2001.

29. Buckwalter JA and Mankin HJ: Articular cartilage: Tissue design and chondrocyte-matrix interactons. Instr Course Lect 47 477-486, 1998

30. Tew SR, Kwan AP, Hann A, Thomson BM and Archer CW: The reactions of articular cartilage to experimental wounding: Role of apoptosis. Arthritis Rheum 43: 215-225, 2000.
31. Hwang HS and Kim HA. Chondrocyte apoptosis in the pathogenesis of osteoarthritis. Int J Mol Sci 16: 26035-26054, 2015.

32. Gu YT, Chen J, Meng ZL, Ge WY, Bian YY, Cheng SW, Xing CK, Yao JL, Fu J and Peng L: Research progress on osteoarthritis treatment mechanisms. Biomed Pharmacother 93: 1246-1252, 2017.

33. Liao Y, Li X, Li N and Zhou J: Electroacupuncture protects against articular cartilage erosion by inhibiting mitogen-activated protein kinases in a rat model of osteoarthritis. Acupunct Med 34: 290-295, 2016

34. Qin Y, He J, Xia L, Guo H and He C: Effects of electro-acupuncture on oestrogen levels, body weight, articular cartilage histology and MMP-13 expression in ovariectomised rabbits. Acupunct Med 31: 214-221, 2013

35. Tang JB, Sheng XP and Fan TY: Study on the effect of electroacupunture on knee joint chondrocyte apoptosis in rabits with knee osteoarthritis. J Tradit Chin Orthop Traumat 24: 12-15, 2012. (In Chinese).

36. Kapuscinski J: DAPI: A DNA-specific fluorescent probe. Biotech Histochem 70: 220-233, 1995.

37. Verhoven B, Schleqel RA and Williamson P: Mechanisms of phosphatidylserine exposure, a phagocyte recognition signal, on apoptotic T lymphocytes. J Exp Med 182: 1597-1601, 1995.

38. Koopman G, Reutelinqsperger CP, Kuijten GA, Keehnen RM, Pals ST and van Oers MH: Annexin V for flow cytometric detection of phosphatidylserine expression on B cells undergoing apoptosis. Blood 84: 1415-1420, 1994.

39. Lecoeur H: Nuclear apoptosis detection by flow cytometry: Influence of endogenous endonucleases. Exp Cell Res 277: 1-14, 2002.

40. Brüne B: Nitric oxide: NO apoptosis or turning it ON?. Cell Death Differ 10: 864-869, 2003.

41. Reers M, Smiley ST, Mottola-Hartshorn C, Chen A, Lin M and Chen LB: Mitochondrial membrane potential monitored by JC-1 dye. Methods Enzymol 260: 406-417, 1995.

42. Robertson JD, Zhivotovsky B, Goqvadze V and Orrenius S: Outer mitochondrial membrane permeabilization: An open-and-shut case? Cell Death Differ 10: 485-487, 2003.

43. Youle RJ and Strasser A: The BCL-2 protein family: Opposing activities that mediate cell death. Nat Rev Mol Cell Biol 9: 47-59, 2008.

44. Hardwick JM and Soane L: Multiple functions of BCL-2 family proteins. Cold Spring Hard Perspect Biol 5: a008722, 2013.

45. Westphal D, Kluck RM and Dewson G: Building blocks of the apoptotic pore: How Bax and Bak are activated and oligomerize during apoptosis. Cell Death Differ 21: 196-205, 2014.

46. Vakifahmetoqlu-Norberq H, Ouchida AT and Norberq E: The role of mitochondria in metabolism and cell death. Biochem Biophys Res Commun 482: 426-431, 2017.

This work is licensed under a Creative Commons Attribution-NonCommercial-NoDerivatives 4.0 International (CC BY-NC-ND 4.0) License. 